\title{
Overexpression of Peroxisome-Localized GmABCA7 Promotes Seed Germination in Arabidopsis thaliana
}

\author{
Jianchun Li ${ }^{1}$, Zaihui Peng ${ }^{1}$, Yan Liu ${ }^{1}$, Meirong Lang ${ }^{1}$, Yaohui Chen ${ }^{1}$, Huihong Wang ${ }^{1}$, Yingshuang Li ${ }^{1}$, \\ Banruo Shi ${ }^{1}$, Weipeng Huang ${ }^{1}$, Li Han ${ }^{1}$, Yifeng Ma ${ }^{1}$, Yu Zhang ${ }^{2}$ and Bangjun Wang ${ }^{1, *}$
}

1 Chongqing Key Laboratory of Plant Resource Conservation and Germplasm Innovation, Key Laboratory of Eco-Environments in Three Gorges Reservoir Region (Ministry of Education), College of Life Sciences, Southwest University, Chongqing 400715, China; ybxyli@163.com (J.L.); zaihpeng@foxmail.com (Z.P.); 13271925385@163.com (Y.L.); lmr1902379220@163.com (M.L.); zwjh2017@126.com (Y.C.); huihongwang@163.com (H.W.); yingshuangli2020@163.com (Y.L.); 13320353872@163.com (B.S.); huangweipengswu@163.com (W.H.); hanlimango@163.com (L.H.); ma_yifeng8803@126.com (Y.M.)

2 College of Food Science, Southwest University, Chongqing 400715, China; y000063@swu.edu.cn

* Correspondence: bangjunwang@swu.edu.cn; Tel.: +86-23-6825-3235

\section{check for} updates

Citation: Li, J.; Peng, Z.; Liu, Y.; Lang, M.; Chen, Y.; Wang, H.; Li, Y.; Shi, B.; Huang, W.; Han, L.; et al.

Overexpression of

Peroxisome-Localized GmABCA7

Promotes Seed Germination in

Arabidopsis thaliana. Int. J. Mol. Sci. 2022, 23, 2389. https://doi.org/ $10.3390 /$ ijms 23042389

Academic Editors: Eva Stoger and Elsa Arcalis

Received: 31 December 2021 Accepted: 18 February 2022 Published: 21 February 2022

Publisher's Note: MDPI stays neutral with regard to jurisdictional claims in published maps and institutional affiliations.

Copyright: (C) 2022 by the authors. Licensee MDPI, Basel, Switzerland. This article is an open access article distributed under the terms and conditions of the Creative Commons Attribution (CC BY) license (https:// creativecommons.org/licenses/by/ $4.0 /)$.

\begin{abstract}
Peroxisome is one of the important organelles for intracellular lipid metabolism in plant cells and $\beta$-oxidation of fatty acids in peroxisomes provides the energy for oil-containing seed germination. In this study, we identified an ATP-binding cassette (ABC) transporter gene, GmABCA7 from soybean, which is highly expressed in the different developmental stages of seeds. Transient expression of GmABCA7 in tobacco epidermal cells showed that GmABCA7 was specifically localized at the peroxisomes. Overexpression of $G m A B C A 7$ in Arabidopsis does not change seed phenotypes, or the overall levels of lipid, protein and sugar stored in the seeds; however, the transgenic seeds produced more gluconeogenic pathway precursors such as succinate and malate and germinated earlier compared to the wild type seeds. These results suggest that GmABCA7 may affect the $\beta$-oxidation of fatty acids and play an important role in seed germination.
\end{abstract}

Keywords: peroxisome; $\beta$-oxidation; seed germination; GmABCA7; soybean

\section{Introduction}

Soybean (Glycine max) is a major oilseed crop worldwide that is also rich in proteins. The oil in soybean seeds is mainly in the form of triacylglycerol (TAG), providing a carbon source and energy for seed germination and seedling establishment. During germination and seedling development, TAG breaks down into fatty acids, which are then converted to succinate in peroxisomes through fatty acid $\beta$-oxidation and glyoxylate cycles, and it ultimately produces sucrose through the gluconeogenic pathway [1]. Thus, plant peroxisomes are the central organelles for storage lipid degradation and are critical for seed germination. Fatty acids can enter a peroxisome with the help of a variety of transporters like the ATP-binding cassette $(\mathrm{ABC})$ transporter AtABCD1 [2].

$\mathrm{ABC}$ transporters are an important class of transmembrane proteins that are widely present in organisms, and their structures and functions are highly conserved [3]. The Arabidopsis genome encodes more than $100 \mathrm{ABC}$ transporters [4,5]. Based on the transmembrane domains (TMDs) and the nucleotide-binding folds (NBFs), all plant $\mathrm{ABC}$ proteins are divided into eight major subfamilies, namely, A, B, C, D, E, F, G and I [4,6,7]. Most ABC transporters are located at the organelle membranes, and are involved in the transport of many substances, including hormones, heavy metal complexes, lipids, and glycosides in Arabidopsis [4].

The AtABCD1/PXA1/PED3/CTS1 is a peroxisome-localized ABC transporter and is involved in some metabolic and developmental processes including seed germination and seedling growth in Arabidopsis [2,4,8]. AtLACS6/7 and AtCGI-58 can physically interact 
with AtABCD1 to facilitate substrates into the $\beta$-oxidation pathway and to co-regulate lipid homeostasis and signaling [8,9]. Seed germination promoted by AtABCD1 could be induced by pectin degradation under the control of ABI5 [10].

In this study, we cloned a new ABC transporter gene, GmABCA7 (GenBank accession: MH910628), from the soybean cultivar, Heinong 44, which encodes a protein of 954 amino acid residues. Currently, only AtABCA9 has been functionally identified from the A subfamily of Arabidopsis ABC transporters, which facilitates seed lipid synthesis at the endoplasmic reticulum [7,11]. GmABCA7 is localized at the peroxisome, suggesting GmABCA7 may have a different function compared to AtABCA9. The succinate and malate levels in the GmABCA7-overexpressing Arabidopsis seeds showed a marked increase compared to those in the wild type. Our data showed that the overexpression of GmABCA7 may affect $\beta$-oxidation of fatty acids and promote seed germination in Arabidopsis, which provides a new strategy to cultivate high-vigorous seeds to increase crop yield.

\section{Results}

\subsection{Structural Features of GmABCA7 and Its Gene Expression}

We cloned a new ABCA transporter gene from soybean, which encodes 954 amino acids (GenBank accession number: MH910628). A homology search against the Arabidopsis genome identified the highest homology with $A t A B C A 7$, thus the gene was named GmABCA7 (Supplementary Figure S1). GmABCA7 had a 72.6\%, 66.9\%, 63.3\%, $57.5 \%$ and $58.9 \%$ amino acid sequence identity to AhABCA7 (XP_025701693), RcABCA7 (XP_015579513), AtABCA7 (NP_190362), ZmABCA7 (XP_008652604) and OsABCA7 (XP_015651056), respectively (Figure 1A).

A

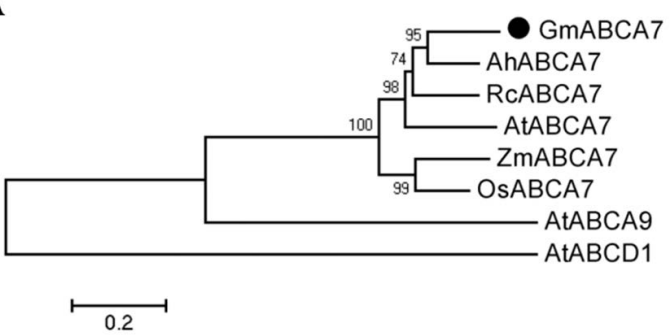

B

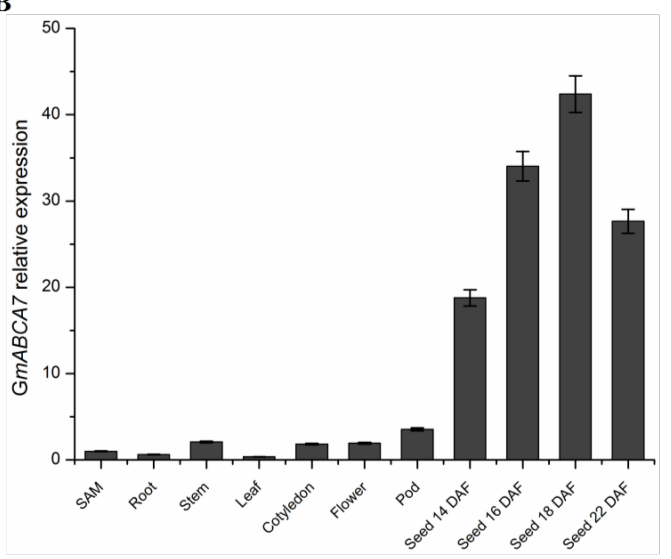

Figure 1. Phylogenetic analysis of GmABCA7 and its expression patterns in different tissues from Glycine max. (A) Phylogenetic tree analysis of GmABCA7 protein and other homologs from Glycine max (GmABCA7, MH910628); Arabidopsis thaliana (AtABCA7, NP_190362; AtABCA9, NP_200981; AtABCD1, NP_001328232); Arachis hypogaea (AhABCA7, XP_025701693); Ricinus communis (RcABCA7, XP_015579513); Oryza sativa (OsABCA7, XP_015651056) and Zea mays (ZmABCA7, XP_008652604). (B) Expression analysis of GmABCA7 in different soybean tissues by qRT-PCR. Roots, stems, SAMs and leaves from two-week seedlings, opening flowers and pods from adult plants, and seeds at 14, 16,18 and 22 DAF were collected for analysis. 
Quantitative RT-PCR (qRT-PCR) data showed that the GmABCA7 was expressed in various tissues (SAM, root, stem, leaf, flower, cotyledon, pod and seeds at different developmental stages) of soybean, with the highest expression in seeds (Figure 1B). The gene was highly expressed between 14 and 22 DAF (Days after flowering) and peaked at $18 \mathrm{DAF}$ in the developing seeds (Figure 1B). These results suggest that GmABCA7 might be involved in the regulation of seed development in soybean.

\subsection{GmABCA7 Is Localized at the Peroxisomes}

To investigate the function of the GmABCA7 transporter, the full-length GmABCA7 cDNA was ligated into the PCX-DR vector to generate the Pro35S-RFP-GmABCA7 and Pro35S-YFP-GmABCA7 vector (Figure 2A). We examined the subcellular localization of GmABCA7 by transiently expressing Pro35S-RFP-GmABCA7 in leaves of Nicotiana benthamiana. A punctate fluorescence pattern was observed in the tobacco leaf epidermal cells, which was completely different from the control (Figure 2B). The punctate fluorescence was similar to the identified peroxisome signals $[12,13]$.

$\mathbf{A}$

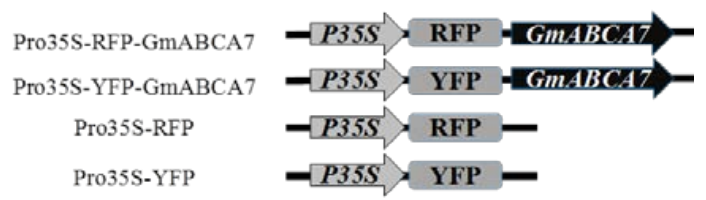

B

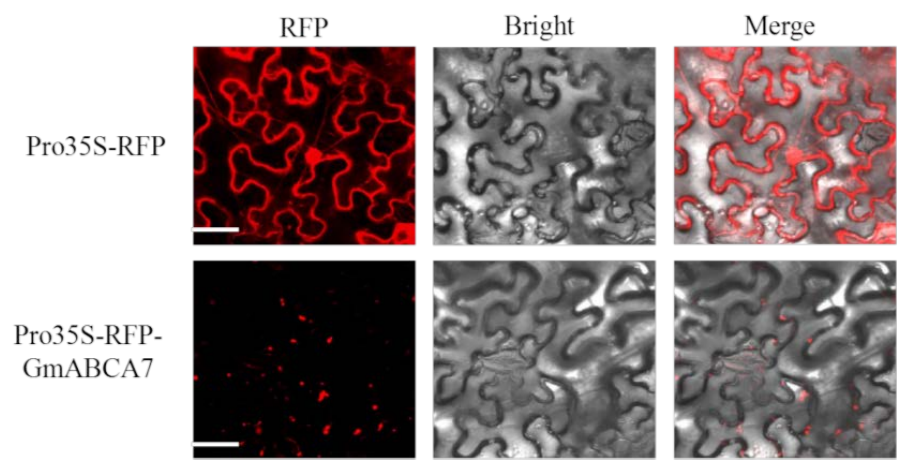

C

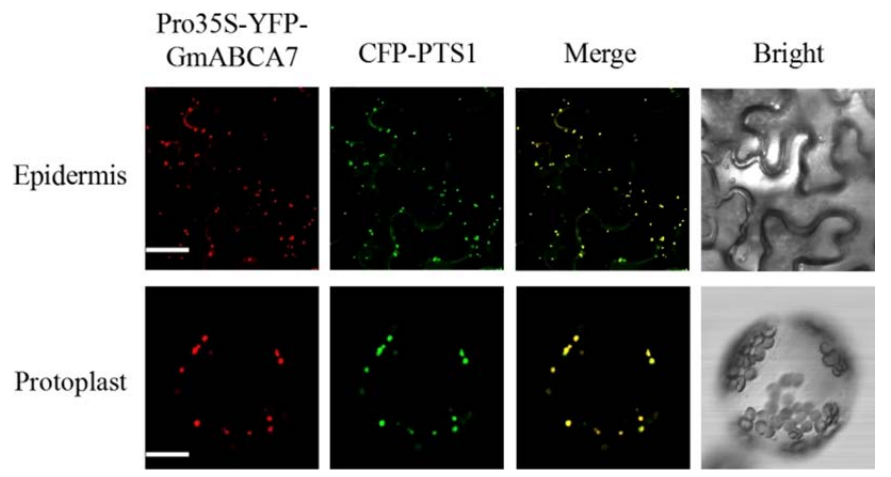

Figure 2. Subcellular localization of GmABCA7 in leaf epidermal cells of Nicotiana benthamiana. (A) Diagram of the Pro35S-RFP-GmABCA7, Pro35S-YFP-GmABCA7, Pro35S-RFP and Pro35S-YFP vectors. (B) Pro35S-RFP-GmABCA7 and Pro35S-RFP were expressed in tobacco epidermal cells respectively. Pro35S-RFP was used as a control. Bars $=50 \mu \mathrm{m}$. (C) Co-expression of Pro35S-YFPGmABCA7 with CFP-PTS1. CFP-PTS1 was used as a marker for peroxisome. Protoplasts were from tobacco leaves co-expressing with CFP-PTS1 and YFP-GmABCA7. RFP, red fluorescent protein. YFP, yellow fluorescent protein. CFP, cyan fluorescent protein. Bars $=20 \mu \mathrm{m}$. 
To further identify the subcellular localization of the GmABCA7 protein, we transiently co-expressed Pro35S-YFP-GmABCA7 with a CFP-PTS1-labeled peroxisomal marker ( $\mathrm{px}$ ck) [14] in tobacco leaves. Co-expression of the Pro35S-YFP-GmABCA7 and CFP-PTS1 clearly showed that the YFP and CFP signals completely overlapped in the leaf epidermal cells and leaf protoplasts (Figure 2C). These results suggest that GmABCA7 is localized at the peroxisomes.

\subsection{Overexpression of GmABCA7 Promotes Seed Germination in Arabidopsis}

Three Arabidopsis transgenic lines (OE-1, OE-3 and OE-4) were examined for GmABCA7 gene expression (Supplementary Figure S2A). To analyze the function of the GmABCA7 during seed development, we examined the transgenic lines in terms of seed-related traits. Developing seeds at 14 DAF and dry seeds from wild type and GmABCA7-overexpressing plants were observed under stereomicroscope. We found that there was no significant difference in seed size and 1000-seed weight between the transgenic lines and the wild type seeds (Figure 3A,B and Supplementary Figure S2B).

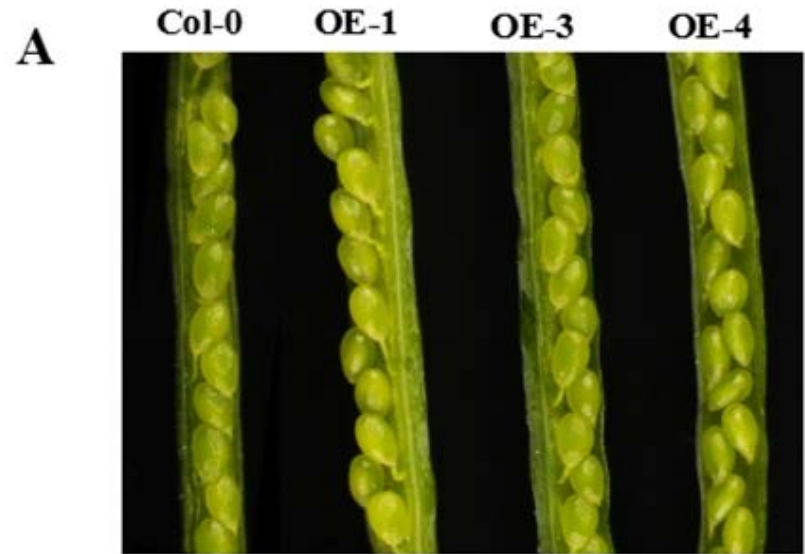

C

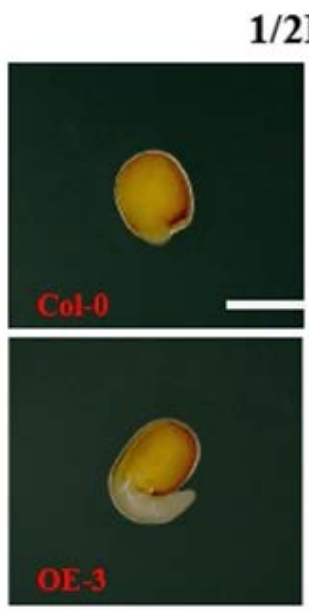

\section{1/2MS}

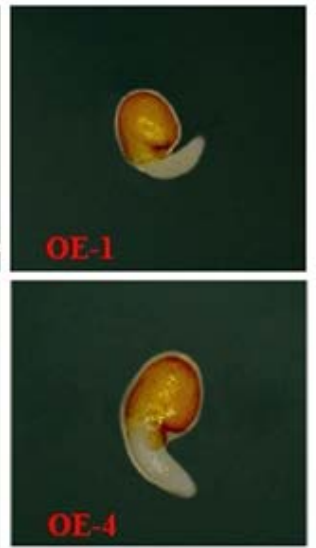

B

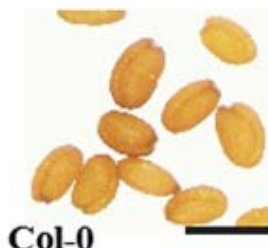

Col-0

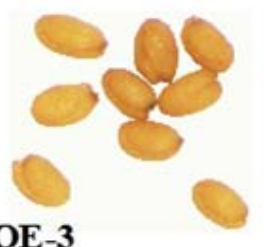

OE-3

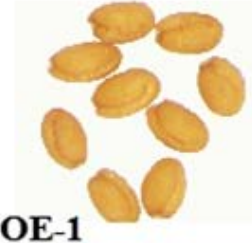

OE-1

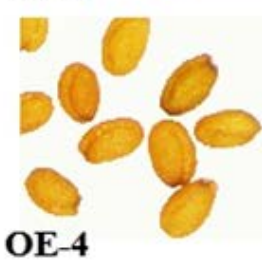

D

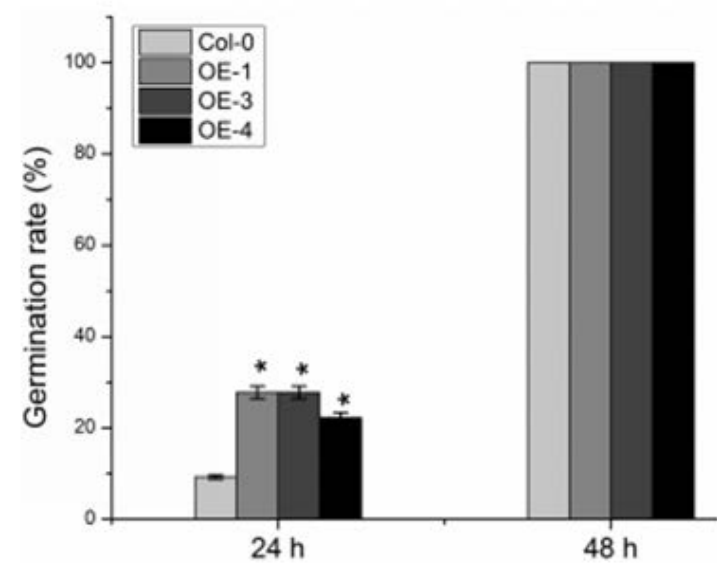

Figure 3. Overexpression of $G m A B C A 7$ promoted seed germination in Arabidopsis. (A) Siliques at 12 days after flowering (12 DAF). (B) Seeds of the wild type and three transgenic lines (OE-1, OE-3 and OE-4). Scale bar $=500 \mu \mathrm{m}$. (C) Seed germination at $24 \mathrm{~h}$ after sowing on $1 / 2 \mathrm{MS}$ medium. Scale bar $=0.5 \mathrm{~mm}$. (D) Germination rates of wild type and $G m A B C A 7$ transgenic seeds were measured $24 \mathrm{~h}$ and $48 \mathrm{~h}$ after sowing on 1/2 MS medium. The wild type and GmABCA7 transgenic seeds (OE-1, OE-3 and OE-4) were grown with a photoperiod of $16 \mathrm{~h}, 22^{\circ} \mathrm{C} / 8 \mathrm{~h}, 20^{\circ} \mathrm{C}$ (light/dark, respectively). Values are means $\pm \mathrm{SE}$ of three independent experiments each using 18 seeds. Asterisks indicate a significant difference compared to the corresponding controls $\left({ }^{*} p<0.05\right)$. 
Lipid, sugar, starch and protein are the main storage substances in seeds. To determine whether overexpression of $G m A B C A 7$ affects the seed storage substances, we examined the lipid, sugar, starch and protein content of the GmABCA7 transgenic lines (Supplementary Figure S2C-F). The overexpression of GmABCA7 had no significant effects on the accumulation of the overall lipid, sugar and protein, except for increased C18:3 fatty acid contents in the Arabidopsis seeds.

To investigate the effect of overexpression $G m A B C A 7$ on seed germination in Arabidopsis, the wild type and GmABCA7 transgenic seeds were grown on 1/2 MS medium, and the radicle protrusion was studied. We found that about $20 \%$ of seeds from the three transgenic lines had prominent radicles at $24 \mathrm{~h}$ after sowing, while much less in the wild type seeds; germination rates of the transgenic lines were $12.96 \%$ to $18.52 \%$ higher compared to the wild type (Figure 3C,D). All the seeds germinated at $48 \mathrm{~h}$ after sowing (Figure 3D). These results suggest that the overexpression of GmABCA7 promoted seed germination in Arabidopsis.

The plant hormones abscisic acid (ABA) and gibberellin (GA) play important roles in the regulation of seed dormancy and the germination process. We studied the germination rates of seeds treated with exogenous ABA and GA. We found that germination rates of the transgenic seeds were higher than that of the wild type seeds upon $0.3 \mu \mathrm{M}, 0.5 \mu \mathrm{M}$ and $1.0 \mu \mathrm{M}$ of the ABA treatments (Supplementary Figure S3A). Germination rates of the transgenic seeds were also significantly higher than that of the wild type upon $0.3 \mu \mathrm{M}$, $0.5 \mu \mathrm{M}$ and $1.0 \mu \mathrm{M}$ of the GA3 treatment (Supplementary Figure S3B). These results suggest that ABA and GA3 might not change the earlier germination phenotype of GmABCA7transgenic seeds compared to wild type seeds.

\subsection{Overexpression of GmABCA7 Increased the Levels of Gluconeogenic Pathway Precursors during Seed Germination}

Fatty acids are metabolized by $\beta$-oxidation to acetyl-CoA [15], which is converted to succinate through a cycle of the glyoxylic acid [16]. Succinate moves into the mitochondrion and is converted to malate, which is then turned into sucrose through the gluconeogenic pathway, providing the carbon source and energy required for germination. To further investigate the function of $G m A B C A 7$ during seed development and germination, we examined levels of the gluconeogenic pathway precursors. The levels of succinate and malate in mature dry seeds were significantly higher compared to the wild type (Figure 4A,B). These results suggest that the GmABCA7 had a positive impact on the $\beta$-oxidation process during seed development, thereby promoting the accumulation of succinate and malate in the seeds. Then, we soaked the seeds with distilled water at $37^{\circ} \mathrm{C}$ for $72 \mathrm{~h}$ to initiate seed germination. The succinate and malate levels in the GmABCA7 overexpression lines were significantly up-regulated (Figure $4 \mathrm{C}, \mathrm{D}$ ), probably due to the highly expressed $G m A B C A 7$ during germination in the transgenic lines (Supplementary Figure S4A).

As previously reported, $\beta$-oxidation could convert 2,4-dichlorophenoxybutyric acid (2,4-DB) into 2,4-dichlorophenoxyacetic acid (2,4-D), and the plants showed stunted root growth when treated with 2,4-DB [17]. GmABCA7 transgenic seedlings were more sensitive to 2,4-DB compared to the wild type (Supplementary Figure S5), suggesting GmABCA7 might enhance $\beta$-oxidation during germination.

These results suggest that the overexpression of $G m A B C A 7$ might increase the levels of gluconeogenic pathway precursors via enhanced $\beta$-oxidation during seed germination in transgenic Arabidopsis. 
A

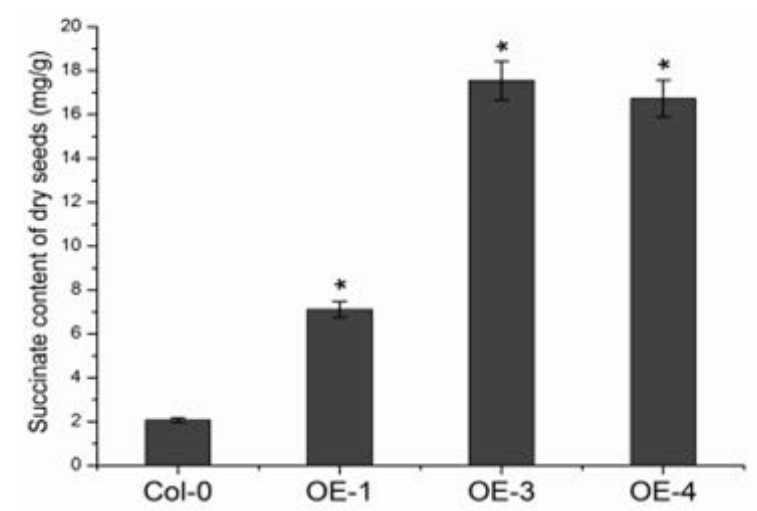

C

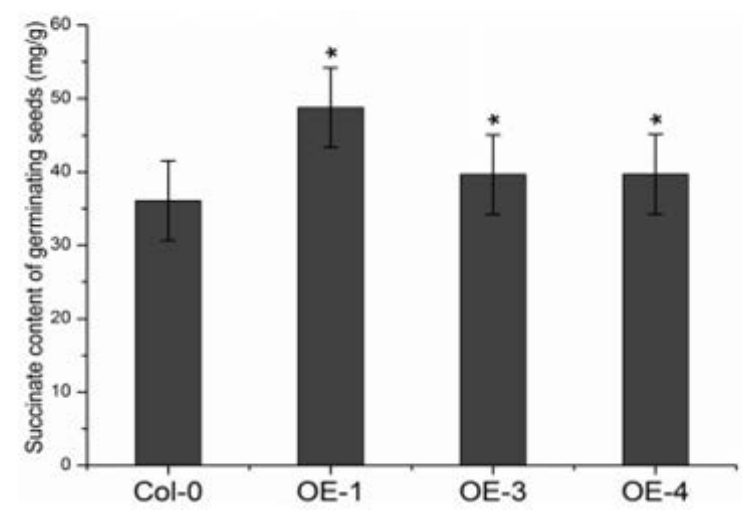

B

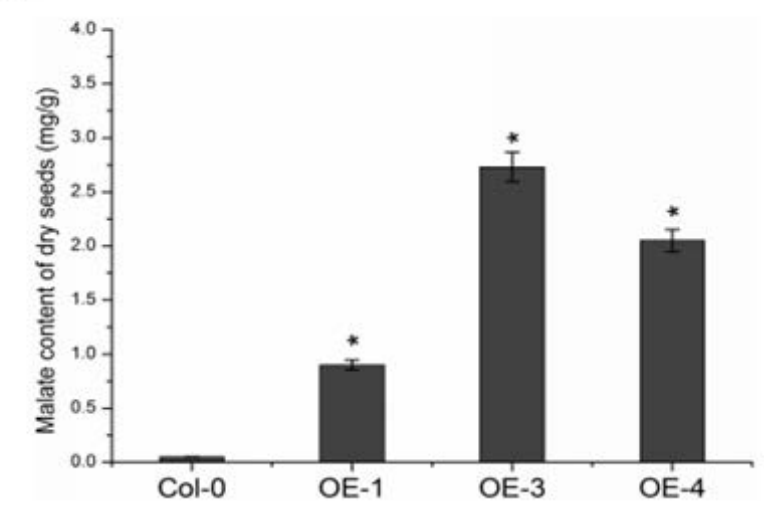

D

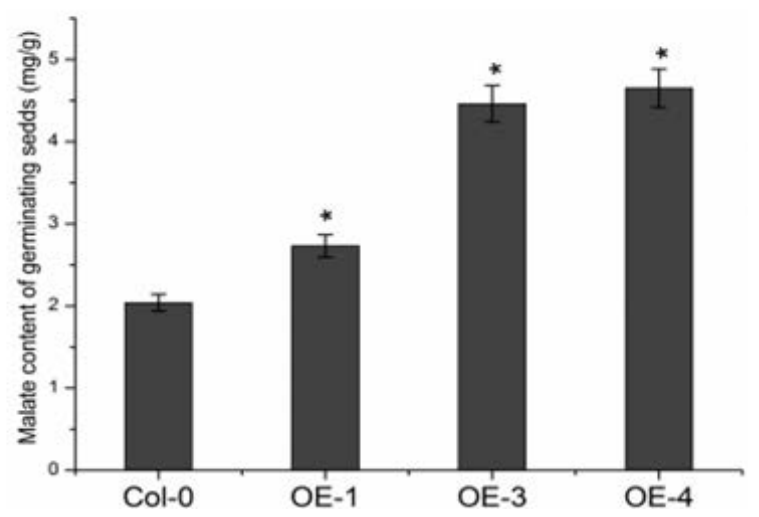

Figure 4. Succinate and malate levels in dry and germinating seeds. (A,B) Succinate and malate levels in mature dry seeds. (C,D) Succinate and malate levels in germinating seeds. Asterisks indicate a significant difference compared to the corresponding controls $\left({ }^{*} p<0.05\right)$. Error bars indicate SEs from three replicates.

\section{Materials and Methods}

\subsection{Bioinformatic and Phylogenetic Analysis}

The structure of the GmABCA7 protein was analyzed using an online website (http:/ / smart.embl-heidelberg.de) on 2 July 2019 [18]. The relevant homologous protein sequences were downloaded from Phytozome (phytozome.jgi.doe.gov) on 2 July 2019 and NCBI (http: / / www.ncbi.nlm.nih.gov / Blast.cgi) on 2 July 2019, and were aligned using DNAMAN 8.0 (Lynnon Biosoft, San Ramon, CA, USA). The phylogenetic tree was constructed using the neighbor-joining (N-J) method provided by the MEGA software v. 5.0 [19].

\subsection{Plant Materials and Growth Conditions}

The soybean cultivar, Heinong 44, provided by the Institute of Genetics and Developmental Biology, Chinese Academy of Sciences, was grown at $25^{\circ} \mathrm{C}$ with a photoperiod of $14 \mathrm{~h} / 10 \mathrm{~h}$ (light/dark, respectively). The soybean root, stem, leaf, flower, pod, shoot apical meristem (SAM), cotyledon and seeds from different developmental stages $(14,16,18$ and 22 Days after flowering, DAF) were kept at $-70{ }^{\circ} \mathrm{C}$ for subsequent RNA isolation.

Arabidopsis thaliana seeds of wild type (ecotype Col-0) and GmABCA7 transgenic plants (OE-1, OE-3 and OE-4) were grown with a photoperiod of $16 \mathrm{~h}, 22{ }^{\circ} \mathrm{C} / 8 \mathrm{~h}, 20^{\circ} \mathrm{C}$ (light/dark, respectively). Seeds were harvested and stored at room temperature for about $2-4$ weeks for the germination test. 


\subsection{RNA Isolation and Quantitative RT-PCR ( $q R T-P C R$ ) Analysis}

Total RNA was isolated from the soybean tissues and Arabidopsis transgenic siliques using BIOZOL Total RNA Extraction Reagent (Bioer Technology, Hangzhou, China) and the cDNA was synthesized using the cDNA synthesis kit (Takara, Japan), according to the manuals. For the qRT-PCR, the Hieff ${ }^{\mathrm{TM}}$ qPCR SYBR ${ }^{\circledR}$ Green Master Mix (High Rox Plus) (Yeasen, Shanghai, China) and the Applied Biosystems StepOne system (Thermo Fisher Scientific, Waltham, MA USA) were used. The GmCYP2.1 and AtTIP41 genes were used as internal controls for the soybean and Arabidopsis, respectively [20,21]. Primers are listed in Supplementary Table S1.

\subsection{Plasmid Construction and Plant Transformation}

The coding sequence of $G m A B C A 7$ (accession number: MH910628) was ligated into the plant binary vector $\mathrm{pCX}-\mathrm{DR}$ and was under the control of the $35 \mathrm{~S}$ CaMV promoter (GenBank: FJ905223) [22], resulting in the Pro35S-RFP-GmABCA7 vector. The KpnI-35SYFP-KpnI fragment was PCR amplified from PXYK (YFP-PTS1) using the Q5 enzyme (NEB, Beijing). Subsequently, the KpnI-35S-DsRED-KpnI fragments of both the Pro35S-RFPGmABCA7 and pCX-DR were replaced by KpnI-35S-YFP-KpnI, to generate Pro35S-YFPGmABCA7 and Pro35S-YFP, respectively. The KpnI-35S-YFP-KpnI regions of these two vectors were confirmed by sequencing.

Constructs were introduced into an Agrobacterium tumefaciens strain GV3101 by electroporation, and the Arabidopsis plants transformed by floral dipping [23]. The Pro35SYFP-GmABCA7 transgenic T1 plants were selected on a half-strength MS medium with $40 \mathrm{mg} / \mathrm{L}$ of hygromycin and $200 \mathrm{mg} / \mathrm{L}$ of cefotaxime, and further confirmed by PCR. The seeds from the transgenic T1 plants were grown for two more generations to screen for homozygous transgenic lines. The primers used are listed in Supplementary Table S1.

\subsection{Subcellular Localization Analysis}

Each plasmid was introduced into the Agrobacterium tumefaciens GV3101. All Agrobacterium strains were cultured in $5 \mathrm{~mL}$ of LB liquid medium overnight, and then the cells were collected by centrifugation at $4000 \mathrm{rpm}, 4^{\circ} \mathrm{C}$ for $10 \mathrm{~min}$, and was re-suspended in an infiltration buffer (100 $\mu \mathrm{M}$ acetosyringone, $10 \mathrm{mM}$ MES- $\mathrm{KOH}$ and $10 \mathrm{mM} \mathrm{MgCl} 2)$ to an OD600 of 0.6 to 0.8 [24]. The bacteria were infiltrated into tobacco leaves and the leaves were kept for 1 day in the dark, then subsequently under normal lighting $\left(16 \mathrm{~h}, 25^{\circ} \mathrm{C} / 8 \mathrm{~h}, 22^{\circ} \mathrm{C}\right.$, light/dark, respectively) conditions for 2 days. Enzymatic hydrolysis was used to free tobacco mesophyll protoplasts [25], prior to the observation of the GmABCA7 protein subcellular localization using a confocal microscope (Olympus FV1200, Japan). Agrobacterium strains harboring pCX-DR and px-ck (CFP-PTS1, http:/ / www.bio.utk.edu/cellbiol/markers/, accessed on 8 March 2019) were used as the control and maker, respectively [14].

\subsection{Seed Lipid, Fatty Acid, Protein and Sugar Analysis}

The seed lipid was quantified according to the hexane extraction method as described [26]. An amount of $100 \mathrm{mg}$ of dry seeds was ground into hexane, then, the hexane supernatant was collected after being centrifuged at 20,000 rpm for $5 \mathrm{~min}$. The seed lipid content was calculated by the amount of lipid after hexane evaporation.

An amount of $50 \mathrm{mg}$ of seeds was used for the extraction of fatty acids as described [27]. After extraction, the fatty acids were subjected to gas chromatography (GC2010, Shimadzu).

Seed protein content was determined using an automated Kjeldahl method [28]. The ground sample $(100 \mathrm{mg})$ was digested by the addition of $10 \mathrm{~mL}$ sulfuric acid and $3.0 \mathrm{~g}$ of a CuSO4- $\mathrm{K}_{2} \mathrm{SO} 4$ mixture $(\mathrm{g} / \mathrm{g}, 1: 10)$. The nitrogen amount (\%) was calculated by using Kjeldahl K-1100 (Hanon) and then converted to the protein amount (\%) by multiplication by 6.25 . 
An amount of $50 \mathrm{mg}$ of seeds was used for the extraction of sugar as described [27]. The ground seeds were extracted with $80 \%(v / v)$ ethanol at $80{ }^{\circ} \mathrm{C}$ for $40 \mathrm{~min}$. After extraction, the supernatant was used to determine the levels of glucose, sucrose and fructose. The samples after the soluble sugar extraction were used for starch quantification.

\subsection{Succinate and Malate Analysis}

Succinate and malate were extracted from the samples by a variation of the hot water extraction method [29], and the extract was subjected to high performance liquid chromatography analysis (HPLC, Agilent 1100$)$. Ground samples $(0.1 \mathrm{~g})$ were mixed with $1 \mathrm{~mL}$ of deionized water and boiled in a $100{ }^{\circ} \mathrm{C}$ water bath for $1 \mathrm{~h}$. The filtrate was centrifuged $\left(10,000 \mathrm{rpm}\right.$ for $15 \mathrm{~min}$ at $\left.25^{\circ} \mathrm{C}\right)$ and transferred to a new centrifuge tube. Finally, it was filtered through a $0.45 \mathrm{~mm}$ membrane filter prior to the HPLC analysis.

\subsection{Statistical Analysis}

Statistical analysis was performed using Microsoft Excel, and analysis of variance was performed using the SPSS 9.0 program. IBM SPSS Statistics 22 was used for the significance analysis. Different letters represented significant differences $(p<0.05)$. Origin 8.5 was used to map the results.

\section{Discussion}

Oilseed's germination depends on the breakdown of storage lipids. The fatty acids are oxidized in one class of peroxisomes called a glyoxysome. Plant peroxisomes participate in many metabolic and signaling pathways, such as the generation of signaling molecules, detoxification, and responses to abiotic and biotic stresses [30]. The subfamily D of ABC transporters are believed to be required for these substrates to pass through the peroxisome membrane, but the transport mechanism is still unclear.

Up to now, few peroxisomal transporters have been identified in plants. The function of the human peroxisomal $\mathrm{ABC}$ transporters (HsABCD1, HsABCD2 and HsABCD3) was found to transport fatty acyl-CoA [31,32]. Arabidopsis AtABCD1 is the most studied plant peroxisomal $\mathrm{ABC}$ transporter and has been identified for multiple functions, including the transport of the fatty acids, indole-3-butyric acid (IBA) and 12-oxophytodienoic acid (OPDA), into peroxisomes for $\beta$-oxidation [9,31-35]. In this study, co-expression of YFPGmABCA7 with CFP-PTS1 showed that GmABCA7 was localized at the peroxisomes (Figure 2), and that $G m A B C A 7$ was highly expressed in developing soybean seeds (Figure 1). These results suggest that GmABCA7 might regulate the transport of certain substances for $\beta$-oxidation in seeds.

Sugar, starch and lipid metabolism play an important role in plant seed germination. We found that the overexpression of GmABCA7 only affected the seed fructose level in OE-3 and OE-4 but did not significantly change the overall levels of total soluble sugar, starch, lipids and proteins (Supplementary Figure S2C-F). Furthermore, we found that there was no difference in seed size and 1000-seed weight between the GmABCA7-overexpressing lines and wild type (Figure 3A,B and Supplementary Figure S2B). These results suggest that the earlier germination of the transgenic seeds is not due to the accumulation of carbon sources.

Succinate and malate are important gluconeogenic pathway precursors. In this study, we found that $G m A B C A 7$-overexpressing lines significantly enhanced succinate and malate levels compared to the wild type, which suggest that the overexpression of GmABCA7 affected the $\beta$-oxidation during seed germination in transgenic Arabidopsis seeds (Figure 4). GmABCA7 was highly expressed in seeds after imbibition in water, while the AtABCA7 expression did not change much in the wild type seeds, suggesting the GmABCA7 functioned at the germination stages in the transgenic lines (Supplementary Figure S4). Furthermore, GmABCA7 transgenic seedlings were more sensitive to 2,4-DB (Supplementary Figure S5). It is possible that $\mathrm{GmABCA7}$ facilitates the $\beta$-oxidation process during seed development, thereby promoting the accumulation of succinate and malate in seeds. 
In conclusion, the overexpression of $G m A B C A 7$ might affect $\beta$-oxidation in seeds, thus providing more carbon source and energy for seed germination. When GmABCA7 is overexpressed in Arabidopsis, the endogenous AtABCA7 expression might also affect the validation of the GmABCA7 function. Therefore, the function of GmABCA7 needs to be further verified in soybean. Up to now, more and more peroxisome localized proteins such as AtABCD1 and AtCGI-58 have been characterized [2,9]. Our study identified a new $\mathrm{ABCA}$ transporter from soybean, which provides a promising target to increase seed vigor and seed germination for soybean breeding.

Supplementary Materials: The following supporting information can be downloaded at: https: //www.mdpi.com/article/10.3390/ijms23042389/s1.

Author Contributions: Methodology, Z.P., H.W. and Y.Z.; validation, Y.L. (Yingshuang Li); formal analysis, W.H.; investigation, J.L., Y.L. (Yan Liu), M.L., Y.C., B.S., L.H. and Y.M.; data curation, J.L., Y.Z. and B.W.; writing — original draft preparation, J.L.; writing — review and editing, B.W.; project administration, B.W.; funding acquisition, B.W. All authors have read and agreed to the published version of the manuscript.

Funding: This work was supported by the National Natural Science Foundation of China (Grant No. 31571584), the Ministry of Science and Technology of China (Grant No. 2016YFD0100504), the National Natural Science Foundation of China (Grant No. 31370317), and the Natural Science Foundation of Chongqing (Grant No. cstc2013jcyjA80016).

Data Availability Statement: All the data in this study are included in this published article and its Supplementary Material files.

Acknowledgments: We thank Ye Ning for the suggestion of the manuscript and the National Teaching Demonstration Center of Food Science and Engineering, Southwest University for technique support.

Conflicts of Interest: The authors declare that they have no conflict of interest.

\section{References}

1. Eastmond, P.J.; Graham, I.A. Re-examining the role of the glyoxylate cycle in oilseeds. Trends Plant Sci. 2001, 6, 72-78. [CrossRef]

2. Kunz, H.H.; Scharnewski, M.; Feussner, K.; Feussner, I.; Flügge, U.I.; Fulda, M.; Gierth, M. The ABC transporter PXA1 and peroxisomal $\beta$-oxidation are vital for metabolism in mature leaves of Arabidopsis during extended darkness. Plant Cell 2009, 21, 2733-2749. [CrossRef]

3. Higgins, C.F. ABC transporters: From microorganisms to man. Annu. Rev. Cell Biol. 1992, 8, 67-113. [CrossRef] [PubMed]

4. Kang, J.; Park, J.; Choi, H.; Burla, B.; Kretzschmar, T.; Lee, Y.; Martinoia, E. Plant ABC transporters. Arabidopsis Book 2011, 9 , e0153. [CrossRef] [PubMed]

5. Hwang, J.U.; Song, W.Y.; Hong, D.; Ko, D.; Yamaoka, Y.; Jang, S.; Yim, S.; Lee, E.; Khare, D.; Kim, K.; et al. Plant ABC transporters enable many unique aspects of a terrestrial plant's lifestyle. Mol. Plant 2016, 9, 338-355. [CrossRef] [PubMed]

6. Rea, P.A. Plant ATP-binding cassette transporters. Annu. Rev. Plant Biol. 2007, 58, 347-375. [CrossRef]

7. Lefèvre, F.; Boutry, M. Towards identification of the substrates of ATP-Binding cassette transporters. Plant Physiol. 2018, 178, 18-39. [CrossRef]

8. De Marcos Lousa, C.; van Roermund, C.W.; Postis, V.L.; Dietrich, D.; Kerr, I.D.; Wanders, R.J.; Baldwin, S.A.; Baker, A.; Theodoulou, F.L. Intrinsic acyl-CoA thioesterase activity of a peroxisomal ATP binding cassette transporter is required for transport and metabolism of fatty acids. Proc. Natl. Acad. Sci. USA 2013, 110, 1279-1284. [CrossRef]

9. $\quad$ Park, S.J.; Gidda, S.K.; James, C.N.; Horn, P.J.; Khuu, N.; Seay, D.C.; Keereetaweep, J.; Chapman, K.D.; Mullen, R.T.; Dye, J.M. The $\alpha / \beta$ hydrolase CGI-58 and peroxisomal transport protein PXA1 coregulate lipid homeostasis and signaling in Arabidopsis. Plant Cell 2013, 25, 1726-1739. [CrossRef]

10. Kanai, M.; Nishimura, M.; Hayashi, M. A peroxisomal ABC transporter promotes seed germination by inducing pectin degradation under the control of ABI5. Plant J. 2010, 62, 936-947. [CrossRef]

11. Kim, S.; Yamaoka, Y.; Ono, H.; Kim, H.; Shim, D.; Maeshima, M.; Martinoia, E.; Cahoon, E.B.; Nishida, I.; Lee, Y. AtABCA9 transporter supplies fatty acids for lipid synthesis to the endoplasmic reticulum. Proc. Natl. Acad. Sci. USA 2013, 110, 773-778. [CrossRef] [PubMed]

12. Pracharoenwattana, I.; Cornah, J.E.; Smith, S.M. Arabidopsis peroxisomal citrate synthase is required for fatty acid respiration and seed germination. Plant Cell 2005, 17, 2037-2048. [CrossRef] [PubMed]

13. Arai, Y.; Hayashi, M.; Nishimura, M. Proteomic identification and characterization of a novel peroxisomal adenine nucleotide transporter supplying ATP for fatty acid $\beta$-oxidation in soybean and Arabidopsis. Plant Cell 2008, 20, 3227-3240. [CrossRef] [PubMed] 
14. Nelson, B.K.; Cai, X.; Nebenfuhr, A. A multicolored set of in vivo organelle markers for co-localization studies in Arabidopsis and other plants. Plant J. 2007, 51, 1126-1136. [CrossRef] [PubMed]

15. Cooper, T.G.; Beevers, H. $\beta$-oxidation in glyoxysomes from castor bean endosperm. J. Biol. Chem. 1969, 244, 3514-3520. [CrossRef]

16. Beevers, H. Metabolic production of sucrose from fat. Nature 1961, 191, 433-436. [CrossRef]

17. Footitt, S.; Slocombe, S.P.; Larner, V.; Kurup, S.; Wu, Y.; Larson, T.; Graham, I.; Baker, A.; Holdsworth, M. Control of germination and lipid mobilization by COMATOSE, the Arabidopsis homologue of human ALDP. EMBO J. 2002, 21, 2912-2922. [CrossRef]

18. Letunic, I.; Bork, P. 20 years of the SMART protein domain annotation resource. Nucleic Acids Res. 2018, 46, D493-D496. [CrossRef]

19. Tamura, K.; Peterson, D.; Peterson, N.; Stecher, G.; Nei, M.; Kumar, S. MEGA5: Molecular evolutionary genetics analysis using maximum likelihood, evolutionary distance, and maximum parsimony methods. Mol. Biol. Evol. 2011, 28, 2731-2739. [CrossRef]

20. Czechowski, T.; Stitt, M.; Altman, T.; Udvardi, M.K.; Scheible, W.R. Genome-wide identification and testing of superior reference genes for transcript normalization in Arabidopsis. Plant Physiol. 2005, 139, 5-17. [CrossRef]

21. Zeng, W.T.; Chai, C.Y.; Dou, D.L. Selection and validation of reference genes for quantitative RT-PCR analysis in soybean. $J$. Nanjing Agric. Univ. 2015, 38, 787-795.

22. Chen, S.B.; Songkumarn, P.; Liu, J.L.; Wang, G.L. A versatile zero background T-vector system for gene cloning and functional genomics. Plant Physiol. 2009, 150, 1111-1121. [CrossRef]

23. Clough, S.J.; Bent, A.F. Floral dip: A simplified method for Agrobacterium-mediated transformation of Arabidopsis thaliana. Plant J. 1998, 16, 735-743. [CrossRef] [PubMed]

24. Sparkes, I.A.; Runions, J.; Kearns, A.; Hawes, C. Rapid, transient expression of fluorescent fusion proteins in tobacco plants and generation of stably transformed plants. Nat. Protoc. 2006, 1, 2019-2025. [CrossRef] [PubMed]

25. Yoo, S.D.; Cho, Y.H.; Sheen, J. Arabidopsis mesophyll protoplasts: A versatile cell system for transient gene expression analysis Nat. Protoc. 2007, 2, 1565-1572. [CrossRef] [PubMed]

26. Shen, B.; Sinkevicius, K.W.; Selinger, D.A.; Tarczynski, M.C. The homeobox gene GLABRA2 affects seed oil content in Arabidopsis. Plant Mol. Biol. 2006, 60, 377-387. [CrossRef] [PubMed]

27. Song, Q.X.; Li, Q.T.; Liu, Y.F.; Zhang, F.X.; Ma, B.; Zhang, W.K.; Man, W.Q.; Du, W.G.; Wang, G.D.; Chen, S.Y.; et al. Soybean GmbZIP123 gene enhances lipid content in the seeds of transgenic Arabidopsis plants. J. Exp. Bot. 2013, 64, 4329-4341. [CrossRef] [PubMed]

28. Kim, H.K.; Kang, S.T.; Oh, K.W. Mapping of putative quantitative trait loci controlling the total oligosaccharide and sucrose content of Glycine max seeds. J. Plant Res. 2006, 119, 533-538. [CrossRef] [PubMed]

29. Jones, E.C.; Barnes, R.J. Non-volatile organic acids of grasses. J. Sci. Food Agric. 1967, 18, 321-324. [CrossRef]

30. Kaur, N.; Reumann, S.; Hu, J. Peroxisome biogenesis and function. Arabidopsis Book 2009, 7, e0123. [CrossRef]

31. van Roermund, C.W.; Visser, W.F.; Ijlst, L.; Waterham, H.R.; Wanders, R.J. Differential substrate specificities of human ABCD1 and ABCD2 in peroxisomal fatty acid $\beta$-oxidation. Biochim. Biophys. Acta 2011, 1811, 148-152. [CrossRef] [PubMed]

32. van Roermund, C.W.; Ijlst, L.; Wagemans, T.; Wanders, R.J.; Waterham, H.R. A role for the human peroxisome half-transporter ABCD3 in the oxidation of dicarboxylic acids. Biochim. Biophys. Acta 2014, 1841, 563-568. [CrossRef]

33. Zolman, B.K.; Silva, I.D.; Bartel, B. The Arabidopsis pxa1 mutant is defective in an ATP-binding cassette transporter-like protein required for peroxisomal fatty acid $\beta$-oxidation. Plant Physiol. 2001, 27, 1266-1278. [CrossRef]

34. Hayashi, M.; Nito, K.; Takei-Hoshi, R.; Yagi, M.; Kondo, M.; Suenaga, A.; Yamaya, T.; Nishimura, M. Ped3p is a peroxisomal ATP-binding cassette transporter that might supply substrates for fatty acid $\beta$-oxidation. Plant Cell Physiol. 2002, $43,1-11$. [CrossRef] [PubMed]

35. Theodoulou, F.L.; Job, K.; Slocombe, S.P.; Footitt, S.; Holdsworth, M.; Baker, A.; Larson, T.R.; Graham, I.A. Jasmonic acid levels are reduced in COMATOSE ATP-binding cassette transporter mutants. Implications for transport of jasmonate precursors into peroxisomes. Plant Physiol. 2005, 137, 835-840. [CrossRef] [PubMed] 